\title{
MEJORAMIENTO GENÉTICO DEL FRIJOL ROJO Y NEGRO MESOAMERICANO PARA CENTROAMÉRICA Y EL CARIBE 1
}

\author{
Juan C. Rosas ${ }^{2}$, Aracely Castro ${ }^{2}$, Edwin Flores ${ }^{2}$
}

RESUMEN

Mejoramiento genético del frijol rojo y negro mesoamericano para Centroamérica y EI Caribe. La producción de frijol en Centroamérica es llevada a cabo mayormente en laderas de suelos marginales, limitada por diversos factores bióticos y abióticos. Más de 350,000 t de este grano se producen en cerca de 0,5 millones de hectáreas, con rendimientos promedios de $678 \mathrm{~kg} / \mathrm{ha}$. La mayoría de los productores de frijol utilizan bajos insumos y muy pocas fincas están mecanizadas. El autoconsumo es alto; sin embargo, una alta proporción de este grano es comprado por intermediarios. En Honduras, el frijol es el sétimo cultivo en valor económico y es el de mayor rentabilidad entre el maíz, arroz y sorgo. Las dos clases comerciales más importantes en Centroamérica son el frijol rojo y negro pequeños (raza Mesoamérica). El mejoramiento de estos tipos de frijol se ha enfocado en el desarrollo de resistencia a enfermedades (mosaico común, mosaico dorado, antracnosis, mancha angular, roya, mustia hilachosa y bacteriosis común) e insectos (principalmente picudo de la vaina); y tolerancia a baja fertilidad, sequía y calor. Programas adecuados de hibridación, evaluación y selección son usados para el desarrollo de cultivares con resistencia múltiple, alto rendimiento, buena adaptación y aceptación comercial. La ampliación de la base genética se obtiene usando fuentes de germoplasma Andino y Mesoamericano en la etapa de hibridación. La selección simultánea por varios caracteres y pruebas multilocalidades son utilizadas. Los viveros y ensayos de adaptación y rendimiento de líneas avanzadas son distribuídos a países de Centroamérica, México, y El Caribe. Las pruebas y validaciones en fincas y la liberación de variedades, son efectuadas por los programas nacionales de frijol de los países miembros de PROFRIJOL, en colaboración con el Zamorano, CIAT y el Bean/Cowpea CRSP.

\begin{abstract}
Breeding of the black and red Mesoamerican beans for Central America and the Caribbean. Bean production in Central America is mainly a small farm operation on hillside, marginal areas, limited by several biotic and abiotic constraints. More than $350,000 \mathrm{t}$ of beans are produced in nearly 0.5 millions of hectáreas, with a rather low yield average of $678 \mathrm{~kg} / \mathrm{ha}$. The majority of bean producers utilize low inputs and few farms are mechanized. Autoconsumption is rather high; however, a great portion of the beans is sold by intermediaries. In Honduras, beans are the 7th most important crop in economic value, and has the highest economic return among corn, rice and sorghum. The major market classes in Central America are small red and black (race Mesoamerican) beans. Improvement of these bean types is focused in developing resistance to diseases (common mosaic, golden mosaic, anthracnose, angular leaf spot, rust, web blight, and common bacterial blight) and pests (mainly pod weevil); and tolerance to low fertility, drought and heat. Appropriate hybridization and selection programs are utilized for developing multiple resistant, high yielding, well adapted and commercially accepted cultivars. A broad genetic base is accomplished by using Andean and Mesoamerican sources of germplasm in the hybridization stage. Simultaneous selection for various traits and multilocation testing of advanced lines are practiced. Yield and adaptation nurseries and trials of improved lines are distributed to Central America, Mexico, Panama and Caribbean countries. Testing of advanced lines and on-farm validation and varietal release, are carried out by National Bean Programs and institutions, members of the PROFRIJOL regional program, in collaboration with Zamorano, CIAT and the Bean/Cowpea CRSP.
\end{abstract}

\section{INTRODUCCIÓN}

El frijol común (Phaseolus vulgaris) es la principal fuente de proteínas para la mayoría de la población rural, y la urbana de menores ingresos, en Centroamérica y El Caribe. En Centroamérica se producen aproxima- damente 350,000 t de frijol en 0,5 millones de ha, y el consumo per cápita de esta leguminosa de grano es de $11,1 \mathrm{~kg}$ (FAO, 1998). Sin embargo, en algunas zonas rurales este puede ser considerablemente mayor $(20-45 \mathrm{~kg}$ en Honduras) (Rosas et al., 1999a). El frijol es mayormente cultivado por agricultores de pequeña escala; por

\footnotetext{
1 Presentado en la XLVI Reunión Anual del PCCMCA, San Juan, Puerto Rico, 2000. Financiado por Bean/Cowpea CRSP (Donación USAID No. DAN-1310-G-SS-6008- 00) y PROFRIJOL.

2 Programa de Investigaciones en Frijol (PIF), Escuela Agrícola Panamericana, Zamorano, Honduras.
} 
ejemplo, en Honduras más del 70\% de la producción de frijol es generada en fincas menores de dos ha. La mayor parte de la producción se lleva a cabo en terrenos de laderas, con pendientes extremas, suelos marginales, y limitaciones causadas por factores bióticos y abióticos. La mayoría de los productores de frijol hacen uso limitado de insumos y sólo un reducido número de fincas se manejan bajo mecanización; a pesar de que en muchos casos se utiliza tracción animal, los implementos y las técnicas usadas son deficientes.

En Centroamérica el frijol es cultivado en dos épocas; la siembra de primera (fin de mayo/inicio de junio) se realiza al inicio de la estación de lluvias, mayormente en monocultivo y con fines de abastecimiento familiar o venta en mercados locales, e incremento de semi1la. En la siembra de postrera (fin de septiembre/inicio de octubre), la más importante en la región ( $>60 \%$ de la producción total), el frijol es sembrado con frecuencia bajo el sistema de relevo con maíz. Los limitantes bióticos más importantes en la región son el mosaico dorado, la bacteriosis común (Xanthomonas campestris pv. phaseoli) y la mustia hilachosa (Thanatephorus cucumeris) en las zonas bajas; y antracnosis (Colletotrichum lindemuthianum), roya (Uromyces appendiculatus) y mancha angular (Phaeoisariopsis griseola) en zonas intermedias-altas. Las variedades criollas son susceptibles al mosaico común, frecuente en zonas con una precipitación menor a 1,500 msnm. El picudo de la vaina (Trichapion godmani) y los gorgojos de grano (Zabrotes subfasciatus y Acanthoscelides obtectus) son las plagas insectiles que causan mayor daño. Otras limitantes bióticas menos frecuentes en la región son la pudrición gris de la raíz (Macrophomina phaseoli), mancha redonda (Chaetoseptoria welmanii), oidio o mildiú polvoso (Erysiphe polygoni), babosa (Sarasinula plebeia), crisomélidos (Diabrotica spp. y Cerotoma spp.) y lorito verde (Empoasca kraemeri). En los últimos años se ha mencionado la presencia de los virus del mosaico severo (El Salvador y Honduras), del amachamiento (Costa Rica y El Salvador) y de la cepa necrótica del mosaico común (República Dominicana). La sequía y la baja fertilidad de los suelos, principalmente por la deficiencia de $\mathrm{N}$ y $\mathrm{P}$, son los factores abióticos que más afectan al cultivo de frijol en la región. Por otro lado, la falta de adaptación a condiciones de altas temperaturas nocturnas en zonas bajas y/o en ciertas épocas de siembra, limitan la producción o expansión del cultivo; por ello es obvio que éste se concentre entre los 400-1200 msnm, donde predominan temperaturas más favorables $\left(18-24{ }^{\circ} \mathrm{C}\right)$.

La mayoría de los pequeños productores de frijol experimentan un reducido acceso a los mercados, así como al crédito. El autoconsumo de frijol es alto; sin embargo, en Honduras más del 55\% de la producción es vendida a intermediarios o en mercados locales. El frijol es el sétimo cultivo en valor económico en Honduras, y tiene el retorno económico más alto entre los granos básicos, incluyendo al arroz, maíz y sorgo. En la región centroamericana la producción promedio de frijol es de $670 \mathrm{~kg} / \mathrm{ha}$ (FAO, 1998). Sin embargo, con variedades mejoradas y un manejo óptimo del cultivo se han obtenido rendimientos superiores a los $2,000 \mathrm{~kg}-$ /ha; con un manejo recomendado, agricultores pequeños pueden obtener incrementos de más del $50 \%$.

El reducido acceso de los agricultores a semilla de variedades mejoradas, ha sido limitante para la diseminación de estas variedades. La falta de sistemas formales de producción y distribución de semilla, ha llevado a las organizaciones de transferencia de tecnología y de desarrollo (ONGs y OPDs), y a los agricultores mismos, a iniciar programas de producción artesanal de semilla. El nuevo enfoque de esta actividad está dirigido a la facilitación de semilla básica y la capacitación integrada de manejo del cultivo, tecnología postcosecha, y la gestión empresarial mediante procesos de valor agregado (procesamiento) y comercialización (Rosas y Castro, 1999b).

Uno de los mayores logros del Programa de Investigaciones en Frijol (PIF) de la Escuela Agrícola Panamericana, Zamorano, Honduras, ha sido su integración a la red del Programa Regional de Frijol para Centroamérica, México y El Caribe (PROFRIJOL), a través de la cual se colabora con investigadores y extensionistas de instituciones de los 11 países miembros, e investigadores del Bean/Cowpea Collaborative Research Support Program (B/C CRSP) y del Centro Internacional de Agricultura Tropical (CIAT). Los avances en este trabajo son parte de las actividades de mejoramiento de las clases comerciales de grano rojo y negro pequeños (raza Mesoamerica), que representan la mayor área cultivada en Centroamérica y que adicionalmente son importantes en México, Cuba, Haití y República Dominicana. Los resultados provienen en su mayoría de las actividades conducidas en los últimos cuatro años en el mejoramiento de frijol de grano rojo; el mejoramiento de frijol de grano negro se inició recientemente, en 1999.

\section{MATERIALES Y MÉTODOS}

Las actividades de mejoramiento de frijol de grano rojo y negro se llevan a cabo siguiendo un esquema basado en la identificación de factores limitantes; la evaluación de germoplasma; el cruzamiento de progenitores; la evaluación y selección de poblaciones segregantes; viveros y ensayos regionales; y la validación y liberación varietal. 


\section{Identificación de factores limitantes y evaluación de germoplasma}

En esta fase se identifican los factores bióticos y abióticos que afectan la producción de frijol en la región. Las limitantes son clasificadas según su prioridad y predominancia en las regiones productoras de mayor importancia. Luego se identifican los colaboradores potenciales y se define su participación.

Los factores bióticos más relevantes identificados en la región centroamericana son el mosaico dorado, la bacteriosis común, la antracnosis, la mancha angular y la mustia hilachosa; y el picudo de la vaina. La sequía y la baja fertilidad son los factores abióticos más importantes. La baja tolerancia a las altas temperaturas limita el cultivo de frijol en zonas bajas costeras del Pacífico y el Atlántico, o en ciertas localidades en el interior. En los últimos años, los virus del mosaico severo y del amachamiento han sido reportados en localidades aisladas de Centroamérica, y la cepa necrótica del virus del mosaico común en El Caribe. Debido a esto, se ha trabajado en el desarrollo de líneas mejoradas contra estos virus, en colaboración con investigadores del B/C CRSP, CIAT y PROFRIJOL.

El objetivo principal de la evaluación de germoplasma, aunque las fuentes que se evalúan ya hayan sido sugeridas como resistentes, radica en la necesidad de someterlas a las condiciones climáticas y la diversidad patogénica locales. Los resultados de esta evaluación permiten utilizar las fuentes con mejor adaptación y resistencia en los programas de cruzamiento. En estas evaluaciones se pueden emplear diversas técnicas. Para la bacteriosis común se efectúa inoculación artificial con agujas múltiples o con aspersión de inóculo usando bomba de motor. Las evaluaciones de antracnosis y mancha angular pueden ser efectivas asperjando soluciones con tejido infectado recolectado en lotes comerciales. Para la roya se pueden utilizar aspersiones de esporas recolectadas en lotes comerciales, o multiplicadas en plantas susceptibles crecidas en casa de malla o invernadero, proveyéndose la condición de humedad necesaria inmediatamente después de la inoculación. Para las evaluaciones de mosaico dorado, mustia hilachosa y picudo de la vaina, se requieren localidades y épocas donde se presenten infestaciones suficientemente altas de estas plagas (p.e. mosaico dorado en Comayagua, Honduras). Las evaluaciones de algunos factores son garantizadas en aquellas localidades donde se presenta una alta incidencia de la enfermedad (p.e. antracnosis en Puriscal, Costa Rica; y mustia hilachosa en Caisán, Panamá). Para las evaluaciones de sequía se pueden utilizar siembras tardías en la segunda época de cultivo, al final de la temporada de lluvias, o en la épo- ca seca empleando sistemas de riego para el establecimiento de las parcelas.

\section{Cruzamiento de progenitores y selección de pobla- ciones segregantes}

La recombinación de variedades con características comerciales y las fuentes de germoplasma para diversos factores, se realiza mediante cruzamiento artificial de plantas crecidas en invernaderos o en el campo. Cruzas simples, triples, dobles o múltiples son utilizadas para lograr las recombinaciones deseadas. Fuentes de germoplasma de diversas razas de los reservorios Andino y Mesoamericano son utilizadas para ampliar la base genética de las progenies (Singh, 1999). Se hace énfasis en los principios de la selección de gametos, en relación al número de plantas utilizadas para realizar las cruzas. Se cruzan pares de plantas (10-20) de cada combinación y se obtiene un mayor número de semillas $\mathrm{F}_{1}$, especialmente cuando se hacen cruzas múltiples y/o se emplean progenitores de otras razas y reservorios, con características muy diversas. El último cruzamiento de una determinada combinación, se hace utilizando las variedades comerciales (criollas o mejoradas) o líneas élites como progenitores femeninos.

La selección se inicia en la generación $\mathrm{F}_{2}$, aplicándose a plantas individuales o familias dependiendo del método de selección (p.e. pedigrí o gametos). En esta generación se selecciona por caracteres morfológicos e incidencia natural de enfermedades, descartándose las plantas o familias muy pobres. En la $\mathrm{F}_{3}$ se seleccionan las mejores plantas de las mejores familias bajo presión natural y/o artificial de enfermedades (antracnosis, bacteriosis y mancha angular), por valor agronómico (hábito de crecimiento y arquitectura, carga reproductiva en la etapa $\mathrm{R} 8$ y días a madurez) y valor comercial del grano (color, forma y tamaño en la fase de postcosecha); y se cosechan las plantas seleccionadas en compuesto masal. En la $\mathrm{F}_{4} \mathrm{y} \mathrm{F}_{5}$, se continúa la selección por reacción a enfermedades (mosaico dorado y otras), y valor agronómico y comercial, procurando manejar estas generaciones intermedias-avanzadas con bajos insumos. En la $\mathrm{F}_{5}$ se cosechan plantas individuales (selección de gametos) o un compuesto de plantas seleccionadas (pedigrí y otros métodos). En la $\mathrm{F}_{6}-\mathrm{F}_{7}$ se evalúan las líneas seleccionadas en varios ambientes de Honduras y de la región de Centroamérica y El Caribe, bajo una amplia diversidad de limitantes bióticos y abióticos, y se identifican los candidatos para el Vivero de Adaptación Centroamericano (VIDAC) del Sistema de Viveros y Ensayos Regionales (SISTEVER) de PROFRIJOL. En el programa de mejoramiento de frijol de grano rojo también se han empleado métodos de 
selección recurrente (dialelo parcial) y retrocruza, para la incorporación de genes específicos.

\section{Viveros y ensayos regionales}

Cada año se preparan y distribuyen más de 50 viveros VIDAC y ensayos regionales de adaptación y rendimiento (ECAR) del SISTEVER, a los países miembros de PROFRIJOL. Los VIDAC son conformados con las líneas avanzadas que se seleccionan después de un proceso de evaluación de poblaciones segregantes por factores bióticos y abióticos múltiples, incluyendo el valor agronómico y valor comercial del grano. El ensayo ECAR está constituido por 14 líneas avanzadas y dos testigos, uno universal (DOR 364) y uno local (la variedad más cultivada en la localidad o país), empleando parcelas constituídas por cuatro surcos de cinco metros de largo y tres repeticiones. Mediante las evaluaciones del VIDAC y ECAR es factible identificar simultáneamente líneas de amplia adaptación (a ambientes y sistemas de producción diversos), así como aprovechar las interacciones entre el genotipo y el ambiente para seleccionar líneas superiores altamente eficientes para ecosistemas específicos. A través de este sistema de viveros y ensayos regionales, se mantiene un flujo continuo de germoplasma mejorado en la región.

\section{Validación y liberación de variedades}

Los programas nacionales efectúan pruebas y validaciones de líneas avanzadas, seleccionadas del VIDAC y ECAR conducidos en sus respectivos países. El flujo de líneas mejoradas a través del SISTEVER permite la evaluación continúa de nuevos materiales dentro de cada país. La liberación de variedades se realiza después de un proceso extenso de validaciones a nivel de finca, incluyendo aspectos agronómicos, comerciales y de consumo, acompañado de la producción de semilla básica y la descripción de la nueva variedad. Durante los procesos de validación y liberación se efectúan días de campo para dar a conocer las virtudes de la futura variedad.

\section{RESULTADOS Y AVANCES}

\section{Evaluación de germoplasma}

La resistencia a factores bióticos y abióticos múltiples ha sido evaluada en varios grupos de germoplasma incluyendo los viveros VIPADOGEN y VIFURE, y viveros específicos de resistencia a antracnosis, bacterio- sis común, mancha angular, roya, baja fertilidad y sequía, permitiendo confirmar fuentes de resistencia bajo las condiciones agroecológicas y de variabilidad patogénica locales. Entre las principales fuentes de resistencia para el mosaico dorado (gen bgm-1) se encuentran Tío Canela-75, DOR 482, ICTA- Ligero y Turbo III (Cuadro 1). Para la mancha angular, las fuentes andinas G05686 y G06727 han mostrado la mayor resistancia a inóculos de Honduras. Para la roya, las líneas PI181996 y Belmidak RR1 son las más recomendadas. Las líneas VAX 3,4,5 y 6, y Wilk2 son las más resistentes a aislamientos locales de bacteriosis común. Entre las fuentes más tolerantes a baja fertilidad se encuentran G03513 y G21212. Las líneas BAT 477 y V8025 continúan siendo las más tolerantes a sequía. El énfasis en las evaluaciones de germoplasma está dirigido a identificar fuentes diversas de resistencia provenientes de los reservorios Andino y Mesoamericano, que permitan ampliar la base genética en los nuevos cruzamientos.

Cuadro 1. Fuentes de germoplasma para el mejoramiento de frijol rojo y negro Mesoamericano.

\begin{tabular}{ll}
\hline \multicolumn{1}{c}{ Factor } & \multicolumn{1}{c}{ Germoplasma } \\
\hline Mosaico dorado & Tío Canela-75, DOR 482, ICTA Ligero \\
& y Turbo III $($ bgm- $)$ ) \\
Bacteriosis común & VAX 3,4,5 y 6, Wilk 2, Oreol, Sel 1309 \\
Mancha angular & G05686, G06727, G16140, AFR 702, \\
& AFR 703 \\
Mustia hilachosa & MD23-24, G2472 \\
Antracnosis & Michigan DRK $($ Co-1 $)$, G2333 $($ Co-4²) \\
Roya & Belmidak RR-1 (Ur-4 y Ur-11) \\
Baja fertilidad & G03513, G21212 \\
& Sequía BAT 477, V8025, SEQ 5, SEA 13 \\
\hline
\end{tabular}

\section{Cruzamiento de progenitores y selección en pobla-} ciones segregantes

Los cruzamientos de progenitores dependen de las líneas recombinantes que se desean obtener. En primera instancia se manejan separadamente las cruzas para grano rojo de las de grano negro. Las cruzas pueden ser simples, dobles y triples, generalmente de líneas élites y una fuente específica de germoplasma (Cuadro 2).

Las recombinaciones múltiples son cruzas de líneas élites por fuentes de germoplasma de razas andinas y mesoamericanas con resistencia a factores bióticos y/o buena adaptación a estreses abióticos. Las líneas élites son materiales promisorios provenientes de viveros y ensayos regionales, o variedades mejoradas de reciente liberación (p.e. Tío Canela-75, Dorado u otras). Las cruzas múltiples pueden incluir fuentes de resistencia a varios factores y/o más de una fuente para un factor específico. 
Cuadro 2. Alternativas de cruzamientos usados para el desarrollo de líneas de frijol rojo y negro.

\begin{tabular}{ll}
\hline \multicolumn{1}{c}{ Recombinación } & Tipo de Cruza \\
\hline Elite x Elite o Elite x Fuente & Simple \\
Elite x Simple & Triple \\
Simple x Simple & Doble \\
Simple x Triple o Simple x Doble & Múltiple \\
Triple x Múltiple & Múltiple \\
Doble x Múltiple & Múltiple \\
\hline
\end{tabular}

z Cruza múltiple para la recombinación de varios factores y/o varios progenitores para un factor.

En la última cruza de una recombinación múltiple se han usado líneas élites como el progenitor femenino, y la $F_{1}$ de una cruza de varios padres (cruza múltiple donante) como el progenitor masculino. Un ejemplo de poblaciones de grano negro provenientes de cruzamientos efectuados en 1999, para iniciar el desarrollo de líneas mejoradas de esta clase comercial de gran importancia a nivel regional, se presenta en el Cuadro 3. Los cruzamientos iniciales se basan en recombinaciones simples y triples de líneas élites de grano negro, que darán origen a las primeras líneas avanzadas a ser incluídas en el VIDAC del año 2002. Las cruzas programadas para el período 2000-2001 se harán con semilla $\mathrm{F}_{1}$ remanente de estas cruzas simples y triples de negros élites, con líneas élites de grano rojo para introducir el gen bmg-1 de resistencia a mosaico dorado, arquitectura y potencial de rendimiento, y/o con fuentes de resis-

Cuadro 3. Poblaciones de grano negro Mesoamericano desarrolladas en 1999.

\begin{tabular}{ll}
\hline Población & \multicolumn{1}{c}{ Pedigrí } \\
\hline PRF 9931 & DOR 390 // ICTA Ostúa / ICTA Ligero \\
PRF 9932 & DOR 500 // ICTA Ostúa / ICTA Ligero \\
PRF 9933 & ICTA Ligero // VAX 5 / MUS 181 \\
PRF 9934 & Turbo III // VAX 5 / BAT 450 \\
PRF 9935 & ICTA Ligero // V 8025 / G 3513 \\
PRF 9936 & Turbo III // V 8025 / G 3513 \\
PRF 9937 & ICTA Ligero // UCR 55 / Cornell 49242 \\
PRF 9938 & Turbo III // UCR 55 / Cornell 49242 \\
PRF 9939 & ICTA Ligero // UCR 55 / G 3513 \\
PRF 9940 & Turbo III // UCR 55 / G 3513 \\
& \\
BCN 0001 & DOR 390 // UPR 9609-2-2 / TC-75 \\
BCN 0002 & DOR 500 // MD 23-24 / TC-75 \\
BCN 0003 & Turbo III // TC-75 / G 06727 \\
BCN 0004 & Turbo III / DOR 390 \\
BCN 0005 & A 785 / ICTA Ligero \\
BCN 0006 & Negro INIFAP / Turbo III \\
BCN 0007 & Negro Veracruz / ICTA Ligero \\
BCN 0008 & ICTA Ligero / WB 12 \\
BCN 0009 & Turbo III / AL 12 \\
BCN 0010 & ICTA Ligero / DT 12 \\
BCN 0011 & Turbo III / BG 3 \\
\hline
\end{tabular}

tencia a factores críticos como mancha angular y mustia hilachosa.

En la selección en poblaciones segregantes para el desarrollo de líneas mejoradas, se enfatiza en la resistencia a factores bióticos y abióticos múltiples; en caracteres agronómicos deseables incluyendo hábito de crecimiento (indeterminado-arbustivo) y arquitectura de la planta (porte erecto) y madurez temprana-intermedia; amplia adaptación; y buen potencial de rendimiento. Las evaluaciones se manejan utilizando la incidencia natural e inoculaciones artificiales de patógenos causantes de enfermedades, o siembras en localidades con incidencia de los factores limitantes que se desean evaluar, como se describió para las evaluaciones de germoplasma. Un esquema simple de este proceso de evaluación de poblaciones segregantes se presenta en el Cuadro 4.

Siguiendo lo descrito, en 1999-2000 se han evaluado una serie de poblaciones segregantes y líneas avanzadas por factores limitantes múltiples. La mayor parte de los materiales seleccionados que se encuentran en generaciones intermedias a avanzadas son de grano rojo. Sin embargo, se cuenta con un buen número de selecciones de grano negro provenientes de cruzas múltiples, donde se usaron fuentes de germoplasma con este tipo de grano. Otras selecciones de grano negro provienen de cruzamientos realizados en el CIAT (poblaciones $\mathrm{MN}$ ). La secuencia en el avance de estas poblaciones incluyendo el número de materiales evaluados y seleccionados en las épocas de primera y postrera de 1999 (A y B) y bajo riego en el verano del 2000 (X), y los criterios de selección aplicados, se detallan en el Cuadro 5. Las poblaciones en generaciones tempranas e intermedias fueron evaluadas en El Zamorano, Honduras, bajo presión de factores limitantes diversos. En las generaciones avanzadas, se conformaron viveros que son evaluados bajo condiciones diversas en Honduras y algunos países de Centroamérica, en colaboración con los programas nacionales, con el fin de incorporar líneas avanzadas altamente promisorias al vivero VIDAC.

\section{Viveros y ensayos regionales}

La conformación de los viveros y ensayos regionales, VIDAC y ECAR, respectivamente, se ha venido haciendo con base en los resultados de las evaluaciones conducidas en diversas localidades de la región de Centroamérica y El Caribe por investigadores de los países miembros de la red de PROFRIJOL, bajo el siguiente procedimiento. Cada año se renueva el $40-50 \%$ de los materiales del VIDAC y el ECAR. Las nuevas lí- 
Cuadro 4. Esquema de evaluación de poblaciones segregantes de frijol rojo y negro Mesoamericano.

\begin{tabular}{l}
\hline Generación \\
\hline Plantas Individuales \\
$\begin{array}{l}\text { F9-F10 } \\
\text { F7-F8 }\end{array}$
\end{tabular}

neas del VIDAC provienen del vivero de líneas avanzadas (LINAF), seleccionadas con base en evaluaciones de líneas en generaciones avanzadas (F6-F8) en una amplia diversidad de localidades, factores limitantes y sistemas de producción en Honduras y algunos países de Centroamérica. Las nuevas líneas del ECAR provienen del VIDAC, y son aquellas que durante dos años consecutivos han ocupado las primeras posiciones por rendimiento, adaptación, resistencia a enfermedades y valor comercial del grano. Las líneas en el VIDAC y
ECAR se mantienen por un máximo de tres años, si ocupan las posiciones superiores. En los Cuadros 6, 7 y 8 se presentan los resultados de las evaluaciones regionales del LINAF, VIDAC y ECAR de grano rojo efectuadas en 1999, con el fin de destacar las característiscas y los resultados de estos viveros y ensayos.

Las evaluaciones del vivero LINAF-99, conformado por 131 líneas avanzadas y un testigo local sembrado cada 20 líneas, conducido en nueve localidades de

Cuadro 5. Avances en el proceso de selección de poblaciones segregantes de frijol rojo en 1999-2000.

\begin{tabular}{|c|c|c|c|}
\hline & \multicolumn{3}{|c|}{ Epoca } \\
\hline & 1999 A & 1999 B & $2000 X$ \\
\hline \multicolumn{4}{|c|}{ Generaciones avanzadas } \\
\hline $\begin{array}{l}\text { PTC-95 } \\
\text { EAP/PRF/BCH-97 }\end{array}$ & 28 pobl.; 109 F5/F6 & $\begin{array}{l}24 \text { pobl.; } \\
81 \text { F6/F7 (bulk) y } 275 \\
\text { F6/F7 (ind.) }\end{array}$ & 17 pobl.; 141 F7/F8 \\
\hline Criterios de selección ${ }^{\mathrm{Z}}$ & $\mathrm{MH}, \mathrm{RD}, \mathrm{VA}, \mathrm{VC}$ & $\begin{array}{l}\text { AN, MA, RY, MD, } \\
\text { RD, VA, VC }\end{array}$ & $\mathrm{BC}, \mathrm{RD}, \mathrm{VA}, \mathrm{VC}$ \\
\hline \multicolumn{4}{|c|}{ Generaciones intermedias } \\
\hline PRF-98 & 10 pobl.; 1812 F2 & 10 pobl.; 317 F3 & $\begin{array}{l}10 \text { pobl.; } 136 \mathrm{~F} 4 \\
\text { (49 negras) }\end{array}$ \\
\hline Criterios de selección & $\mathrm{MH}, \mathrm{VA}, \mathrm{VC}$ & AN, MA, RY, VA, VC & $\mathrm{BC}, \mathrm{RD}, \mathrm{VA}, \mathrm{VC}$ \\
\hline MR/MN (CIAT 98) & 27 pobl.; 1336 F2 & 24 pobl.; 266 F3 & $\begin{array}{l}24 \text { pobl.; } 144 \mathrm{~F} 4 \\
\text { (80 negras) }\end{array}$ \\
\hline Criterios de selección & MR, MH, BC, VA, VC & AN, MA, RY, MD, VA, VC & $\mathrm{BC}, \mathrm{RD}, \mathrm{VA}, \mathrm{VC}$ \\
\hline \multicolumn{4}{|c|}{ Generaciones tempranas } \\
\hline $\begin{array}{l}\mathrm{BCH} / \mathrm{BC} 3 / \mathrm{PPB}-99 \\
\text { Criterios de selección }\end{array}$ & & $\begin{array}{l}9 \text { pobl.; } \sim 5,000 \mathrm{~F} 2 \\
\text { MA, VA, VC }\end{array}$ & $\begin{array}{l}9 \text { pobl.; } 523 \text { F3 } \\
\text { BC, RD, VA, VC }\end{array}$ \\
\hline $\begin{array}{l}\text { SRC2-99 } \\
\text { Criterios de selección }\end{array}$ & $\begin{array}{l}28 \text { pobl.; } 3,000 \text { F2 } \\
\text { MA, VA, VC }\end{array}$ & & \\
\hline
\end{tabular}


cuatro países Centroamérica (Costa Rica, El Salvador, Nicaragua y Honduras) durante 1999, han permitido identificar materiales promisorios para su incorporación al VIDAC-2000. Como se observa, varias de las 20 líneas superiores por rendimiento incluídas en el Cuadro 6 superan al testigo local, no solamente en rendimiento sino en su valor agronómico (hábito de crecimiento, madurez y carga reproductiva), valor comercial del grano (incluyendo tipo rojo claro como las variedades criollas tradicionales), y mayor resistencia a enfermedades, principalmente mosaico dorado y antracnosis. En cuanto a la madurez, las líneas promisorias fueron similares a los testigos. Entre estas líneas se encuentran materiales generados por los programas de mejoramiento de frijol de Zamorano, la Universidad de Puerto Rico y el CIAT, lo que resalta la importancia de la colaboración entre estos programas en beneficio de la región de Centroamérica y El Caribe. Los resultados de este vivero son alentadores en el sentido de que las líneas promisorias no sólo son superiores a los testigos locales por su mayor potencial de rendimiento y resistencia a enfermedades, sino porque presentan una cali- dad comercial de grano similar a las variedades tradicionales, lo que podría favorecer su adopción por los agricultores debido a la mejor aceptación por los intermediarios o la comercialización directa en mercados locales o regionales. Una maduración similar a la de las variedades locales significa una buena posibilidad de adaptación a los sistemas de producción en la región.

Los resultados del VIDAC-99 en el Cuadro 7 provienen de evaluaciones realizadas en 14 localidades de seis países de Centroamérica y El Caribe (Costa Rica, El Salvador, Haití, Honduras, Nicaragua y Panamá). Se presenta la relación de las mejores 20 líneas ordenadas por rendimiento de un total de 93 más un testigo local (sembrado cada 10 líneas), evaluados por rendimiento, valor agronómico y comercial, y resistencia a varias enfermedades. El promedio de rendimiento de los testigos locales se ubica en la vigésimo octava posición, debido al empleo de variedades mejoradas como testigos locales en algunas de las localidades; sin embargo, las líneas más destacadas superan al promedio de los testigos y presentan un valor agronómico superior a éstos.

Cuadro 6. Resultados de las evaluaciones del vivero de Líneas Avanzadas de Frijol (LINAF-99) en nueve localidades de Centroamérica. 1999.1

\begin{tabular}{|c|c|c|c|c|c|c|c|c|c|c|c|c|}
\hline \multirow{2}{*}{$\begin{array}{c}\mathbf{N}^{\circ} \\
\text { Orden }\end{array}$} & \multirow[b]{2}{*}{ Línea } & \multirow{2}{*}{$\begin{array}{c}\text { Rdto } \\
\text { (kg/ha) }\end{array}$} & \multicolumn{4}{|c|}{ Factores Agronómicos 3} & \multicolumn{6}{|c|}{ Enfermedades 3} \\
\hline & & & DF & DM & VA & $\mathrm{VC}$ & MA & MD & MH & $\mathbf{R Y}$ & AN & MR \\
\hline 1 & MR 12315-86A & 2,939 & 37 & 70 & 5 & 3 & 5 & 3 & 7 & 5 & 7 & 6 \\
\hline 2 & MR 12315-99A-3 & 2,573 & 36 & 70 & 6 & 3 & 6 & 2 & 6 & 5 & 7 & 5 \\
\hline 3 & PRF 9658-84B & 2,569 & 38 & 69 & 5 & 2 & 6 & 3 & 7 & 4 & 6 & 5 \\
\hline 4 & UPR 9806-43-2 & 2,556 & 38 & 71 & 6 & 6 & 5 & 4 & 6 & 5 & 6 & 6 \\
\hline 5 & EAP 9506-14A & 2,505 & 37 & 71 & 6 & 4 & 6 & 2 & 7 & 6 & 3 & 6 \\
\hline 6 & MR 12318-1-9 & 2,416 & 38 & 72 & 6 & 9 & 5 & 5 & 6 & 6 & 4 & 6 \\
\hline 7 & UPR 9808-14-1 & 2,415 & 38 & 69 & 5 & 2 & 7 & 3 & 7 & 4 & 6 & 6 \\
\hline 8 & EAP 9503-41 & 2,401 & 37 & 71 & 5 & 6 & 4 & 3 & 6 & 4 & 7 & 3 \\
\hline 9 & MR 12315-98A-2 & 2,398 & 37 & 70 & 5 & 3 & 6 & 2 & 6 & 5 & 6 & 5 \\
\hline 10 & MR 12322-164B-3 & 2,374 & 37 & 71 & 6 & 3 & 5 & 3 & 7 & 4 & 6 & 6 \\
\hline 11 & PRF 9652-57-1 & 2,352 & 38 & 70 & 6 & 4 & 6 & 3 & 7 & 6 & 8 & 6 \\
\hline 12 & PRF 9651-77-9 & 2,344 & 37 & 70 & 6 & 5 & 5 & 3 & 7 & 5 & 4 & 6 \\
\hline 13 & PTC 9551-9 & 2,340 & 37 & 70 & 5 & 3 & 5 & 4 & 7 & 5 & 4 & 6 \\
\hline 14 & EAP 9504-30A & 2,294 & 38 & 69 & 6 & 3 & 6 & 3 & 6 & 3 & 4 & 6 \\
\hline 15 & PRF 9654-46B-2 & 2,274 & 38 & 71 & 6 & 5 & 6 & 4 & 7 & 5 & 7 & 6 \\
\hline 16 & MR 12315-85B & 2,270 & 37 & 71 & 5 & 2 & 6 & 3 & 7 & 5 & 7 & 6 \\
\hline 17 & EAP 9505-14B & 2,247 & 38 & 70 & 6 & 4 & 5 & 4 & 6 & 2 & 6 & 3 \\
\hline 18 & PRF 9653-16B-2B & 2,238 & 38 & 71 & 6 & 5 & 6 & 3 & 7 & 4 & 5 & 6 \\
\hline 19 & MR 12319-3-7B & 2,236 & 36 & 70 & 6 & 7 & 6 & 4 & 6 & 7 & 5 & 6 \\
\hline 20 & EAP 9506-17B & 2,223 & 38 & 70 & 4 & 5 & 5 & 3 & 7 & 4 & 2 & 3 \\
\hline \multirow[t]{5}{*}{81} & Testigo local 2 & 1,883 & 36 & 69 & 7 & 6 & 6 & 6 & 6 & 4 & 7 & 5 \\
\hline & Promedio & 1,956 & 37 & 70 & 6 & 4 & 6 & 4 & 6 & 5 & 5 & 6 \\
\hline & DMS (\%) & 623 & 1,4 & 3,9 & 1,1 & 2,5 & 1,4 & 1,5 & 1,8 & 3,1 & 3,8 & -- \\
\hline & Localidades & 9 & 5 & 4 & 7 & 2 & 3 & 3 & 2 & 2 & 2 & 1 \\
\hline & $\begin{array}{l}\text { Rango } \\
(\mathrm{n}=131)\end{array}$ & $\begin{array}{l}1,020- \\
2,939\end{array}$ & $33-39$ & $55-72$ & $4-7$ & $2-9$ & $4-7$ & $2-6$ & $4-8$ & $2-8$ & $2-8$ & $2-7$ \\
\hline
\end{tabular}

${ }^{1} \mathrm{DF}=$ días a floración; $\mathrm{DM}=$ días a madurez fisiológica; $\mathrm{VA}=$ valor agronómico; $\mathrm{VC}=$ valor comercial; $\mathrm{MA}=$ mancha angular; $\mathrm{MD}=$ mosaico dorado; $\mathrm{MH}=$ mustia hilachosa; $\mathrm{RY}=$ roya; $\mathrm{AN}=$ antracnosis; $\mathrm{MR}=$ mancha redonda.

2 MD 23-24 en Costa Rica; Desarrural, Charrano y Dorado en Honduras; no se identificaron los testigos en los LINAF-9 conducidos en Nicaragua y Panamá.

3 Escala 1: 10: VA-VC. 
El ECAR es el ensayo más avanzado que resulta del proceso de cruzamiento y selección para el mejoramiento de frijol en la región de Centromérica y El Caribe. Estos ensayos están conformados por 14 líneas avanzadas provenientes de los viveros VIDAC evaluados en años anteriores, e incluyen dos testigos, uno universal y uno local. El ECAR-99 se condujo en 17 localidades de cinco países de la región de Centromérica y El Caribe (Costa Rica, El Salvador, Honduras, Nicaragua y Panamá), y con la participación de la mayoría de los miembros de la red de PROFRIJOL y de otras organizaciones regionales. Las líneas promisorias que los programas nacionales desean validar y liberar en sus países, provienen mayormente de este ensayo.

En el Cuadro 8 se presenta un resumen de los valores promedios obtenidos por las líneas y los testigos en la evaluación regional del ensayo ECAR-99, así como el valor promedio general, rango y separación de medias correspondientes. Los resultados indican que los genotipos con mayor rendimiento tienen un potencial de producción superior al testigo universal (DOR 364) y al promedio del testigo local. Con base en el rendimiento y los coeficientes de estabilidad, donde los valores de $\mathrm{b}=1 \mathrm{y} \mathrm{Sb}=0$ indican mayor estabilidad (Eberhart y Russell, 1966), entre las mejores líneas se encuentran EAP 9510-1, EAP 9508-93 y UPR 9609-2-2. Los coeficientes de estabilidad permiten identificar entre los genotipos más eficientes para condiciones más favorables (o de mejor respuesta a insumos), a las líneas EAP 9510-1 y SRC1-1-18; y entre las más eficientes para ambientes (localidades) menos favorables, a PCE 9351-8 y Dorado. En algunos de los materiales con mejor rendimiento se observa un comportamiento agronómico superior al del testigo local, y un buen avance en el mejoramiento de las líneas por valor comercial del grano, siendo las líneas de mejor rendimiento EAP 9510-1 y EAP 950893, de mejor calidad de grano que los testigos (que incluyen variedades criollas).

Debido a las interacciones significativas genotipoambiente, las evaluaciones individuales de cada locali-

Cuadro 7. Resultados de las evaluaciones del Vivero de Adaptación Centroamericano (VIDAC-99) en 14 localidades de Centroamérica y El Caribe. $1999^{Z}$

\begin{tabular}{|c|c|c|c|c|c|c|c|c|c|c|c|}
\hline \multirow{2}{*}{$\begin{array}{l}\mathrm{N}^{\circ} \\
\text { Orden }\end{array}$} & \multirow[b]{2}{*}{ Línea } & \multirow{2}{*}{$\begin{array}{l}\text { Rdto } \\
\text { (kg/ha) }\end{array}$} & \multicolumn{4}{|c|}{ Factores Agronómicos } & \multicolumn{5}{|c|}{ Enfermedades } \\
\hline & & & DF & DM & VA & VC & MA & MD & MH & $\mathbf{R Y}$ & MS \\
\hline 1 & EAP 9508-41 & 2.144 & 37 & 72 & 5 & 4 & 5 & 3 & 5 & 7 & 4 \\
\hline 2 & PRF 9659-25B-1 & 2.070 & 37 & 72 & 5 & 3 & 6 & 3 & 6 & 7 & 3 \\
\hline 3 & PRF 9653-16B-1 & 2.028 & 38 & 63 & 5 & 4 & 6 & 2 & 7 & 6 & 3 \\
\hline 4 & PRF 9659-35-2 & 1.983 & 35 & 68 & 5 & 4 & 7 & 4 & 6 & 6 & 3 \\
\hline 5 & PRF 9659-35-8 & 1.976 & 35 & 67 & 5 & 4 & 8 & 4 & 7 & 6 & 4 \\
\hline 6 & PTC 9557-10 & 1.894 & 38 & 71 & 5 & 2 & 6 & 4 & 5 & 3 & 3 \\
\hline 7 & PRF 9653-16B-2A & 1.842 & 38 & 71 & 5 & 2 & 5 & 2 & 6 & 6 & 3 \\
\hline 8 & PRF 9657-53-14 & 1.836 & 36 & 71 & 6 & 3 & 5 & 5 & 6 & 4 & 4 \\
\hline 9 & PRF 9657-53-13 & 1.826 & 36 & 72 & 5 & 4 & 7 & 5 & 7 & 6 & 2 \\
\hline 10 & EAP 9508-48 & 1.809 & 38 & 71 & 6 & 5 & 6 & 3 & 6 & 7 & 4 \\
\hline 11 & PRF 9660-79-1 & 1.776 & 38 & 73 & 5 & 5 & 5 & 4 & 5 & 6 & 3 \\
\hline 12 & PRF 9657-53-2 & 1.752 & 36 & 71 & 5 & 3 & 6 & 4 & 6 & 6 & 3 \\
\hline 13 & EAP 9501-7 & 1.747 & 37 & 71 & 6 & 3 & 7 & 2 & 6 & 3 & 6 \\
\hline 14 & PRF 9657-53-1 & 1.744 & 37 & 71 & 6 & 3 & 6 & 3 & 6 & 7 & 4 \\
\hline 15 & EAP 9501-48 & 1.744 & 37 & 72 & 6 & 6 & 7 & 2 & 7 & 6 & 7 \\
\hline 16 & PTC 9558-4 & 1.730 & 36 & 68 & 5 & 5 & 6 & 4 & 6 & 5 & 3 \\
\hline 17 & EAP 9503-35 & 1.721 & 38 & 72 & 5 & 5 & 5 & 3 & 5 & 3 & 6 \\
\hline 18 & RS 4-1 & 1.701 & 38 & 72 & 5 & 5 & 5 & 5 & 6 & 4 & 4 \\
\hline 19 & RS 3-4 & 1.681 & 38 & 71 & 5 & 4 & 5 & 3 & 6 & 2 & 6 \\
\hline 20 & PTC 9558-48 & 1.667 & 37 & 68 & 5 & 5 & 7 & 4 & 6 & 3 & 3 \\
\hline \multirow[t]{5}{*}{28} & Testigo Local y & 1.638 & 36 & 67 & 6 & 3 & 7 & 7 & 7 & 9 & 3 \\
\hline & Promedio & 1.569 & 37.2 & 70,4 & 5,2 & 3,9 & 6,1 & 3,4 & 6,2 & 5,4 & 4,2 \\
\hline & DMS (\%) & 468 & 1,2 & 3,7 & 1,1 & 2,1 & 1,6 & 2,1 & 1,4 & - & - \\
\hline & Localidades & 14 & 7 & 6 & 8 & 3 & 3 & 4 & 3 & 1 & 1 \\
\hline & $\begin{array}{l}\text { Rango } \\
(\mathrm{n}=93)\end{array}$ & $\begin{array}{l}1.053- \\
2.144\end{array}$ & $33-39$ & $63-74$ & $4-6$ & $2-6$ & $4-8$ & $2-7$ & $5-8$ & $2-9$ & $2-8$ \\
\hline
\end{tabular}

z $\mathrm{DF}=$ días a floración; $\mathrm{DM}=$ días a madurez fisiológica; $\mathrm{VA}=$ valor agronómico; $\mathrm{VC}=$ valor comercial; $\mathrm{MA}=$ mancha angular; $\mathrm{MD}=$ mosaico dorado; $\mathrm{MH}=$ mustia hilachosa; $\mathrm{RY}=$ roya; $\mathrm{MS}=$ mosaico severo.

y Chirripó Rojo y Huetar en Costa Rica; Catracho de Seda en El Salvador; Desarrural. Charrano y Dorado en Honduras; DOR 364 en Nicaragua; no se identificaron los testigos en los VIDAC-99 conducidos en Lempa. El Salvador, ni en Panamá. 
Cuadro 8. Rendimiento de grano, coeficientes de estabilidad, valor agronómico, y días a floración y a madurez fisiológica, de las líneas y testigos del ECAR-99 evaluado en 17 localidades de Centroamérica.

\begin{tabular}{lccccccc}
\hline \multicolumn{1}{c}{ Línea } & $\begin{array}{c}\text { Rdto. } \\
\mathbf{( k g / h a )}\end{array}$ & $\begin{array}{c}\text { Coef. Estabilidad* } \\
\mathbf{b}\end{array}$ & $\mathbf{S b}$ & $\mathbf{D F}$ & $\mathbf{D M}$ & VA $^{* * * *}$ & VC \\
\hline EAP9510-1 & 2.259 & 1,17 & 0,07 & 37 & 75 & 6 & 2 \\
EAP 9508-93 & 2.064 & 1,05 & 0,09 & 37 & 75 & 5 & 3 \\
UPR 9609-2-2 & 2.009 & 0,99 & 0,10 & 37 & 73 & 5 & 5 \\
EAP 9510-77 & 1.970 & 1,07 & 0,08 & 37 & 74 & 5 & 4 \\
DICTA 117 & 1.958 & 1,09 & 0,07 & 37 & 72 & 6 & 5 \\
EAP 9509-29 & 1.958 & 1,04 & 0,08 & 37 & 73 & 5 & 4 \\
SRC 1-1-18 & 1.926 & 1,13 & 0,09 & 38 & 74 & 5 & 5 \\
DICTA 146 & 1.890 & 1,03 & 0,07 & 37 & 75 & 6 & 5 \\
PTC 9558-17 & 1.873 & 1,00 & 0,05 & 36 & 73 & 6 & 6 \\
PTC 9557-98 & 1.857 & 0,93 & 0,07 & 37 & 73 & 5 & 4 \\
PM 9422-3 & 1.849 & 0,99 & 0,06 & 37 & 73 & 6 & 5 \\
Test, Local ** & 1.836 & 0,95 & 0,06 & 36 & 72 & 6 & 4 \\
PCE 9351-8 & 1.803 & 0,88 & 0,08 & 38 & 75 & 5 & 4 \\
SRC 1-12-1 & 1.785 & 0,90 & 0,07 & 36 & 72 & 5 & 5 \\
Dorado (T,U, & 1.741 & 0,85 & 0,08 & 38 & 75 & 5 & 5 \\
SRC 1-18-1A & 1.725 & 0,95 & 0,09 & 38 & 75 & 6 & 4 \\
Promedio & 1.907 & - & - & 37 & 74 & 5 & 4 \\
DMS & 183 & - & - & 0,9 & 1,9 & 0,5 & 0,6 \\
Localidades & 15 & 15 & 15 & 10 & 5 & 5 & 3 \\
\hline
\end{tabular}

* $\mathrm{DF}=$ días a floración; $\mathrm{DM}=$ días a madurez fisiológica; $\mathrm{VA}=$ valor agronómico; $\mathrm{VC}=$ valor comercial.

y Coeficientes de estabilidad: regresión (b) y desviación estándar de la regresión (Sb) según Eberhart y Russell (1966).

** Huetar y MD 23-24 en Costa Rica; DOR 585 y Rojo de Seda en El Salvador; Catrachita, Charano, Dorado y Retinto en Honduras; Compañia y DOR 364 en Nicaragua; no se identificaron los testigos en los ECAR-99 conducidos en Puriscal (Costa Rica), Condega (Nicaragua) ni en Panamá.

*** CIAT, 1987

dad indican más claramente las diferencias entre genotipos bajo condiciones específicas de producción. Esto sugiere que para cada país es posible obtener líneas evaluadas, que se incluirían en los ensayos en fincas y/o lotes de validación. Se está considerando la renovación del 50\% de las líneas que forman el ECAR- 2000, por las que han sobresalido en el VIDAC-99.

Con relación a la reacción a enfermedades, las líneas con mejor rendimiento de grano, también presentaron niveles de resistencia aceptables para por lo menos tres enfermedades incluyendo antracnosis, mancha angular, mosaico dorado, mustia hilachosa y roya (Cuadro 9). En este año el testigo universal Dorado, que en años anteriores ha sido difícil de superar, resultó inferior a las mejores líneas y aventajado por el mejor valor comercial del grano de las mismas. Cabe indicar que en algunas localidades se incluyeron como testigos locales algunas variedades mejoradas. En general, se ha avanzado en la identificación de líneas de buen comportamiento, principalmente si se analizan los resultados por localidad en donde algunas de ellas con promedio superior se destacaron con mayor significancia en varios aspectos además del rendimiento.

\section{Validación y liberación de variedades}

En esta fase se concretan los esfuerzos realizados por los programas de mejoramiento de frijol, y se confirman los avances genéticos obtenidos a través de los procesos de hibridación y selección. Con base en el comportamiento en los ensayos ECAR, los programas nacionales conducen actividades de validación varietal en diversas regiones, en colaboración con organizaciones y agricultores interesados. La información obtenida en las validaciones es utilizada para respaldar el proceso de liberación de variedades, que adicionalmente debe estar acompañado de la descripción de la nueva variedad y de la producción de suficiente semilla para su liberación oficial. Se mencionan las variedades de grano rojo Mesoamericano que se encuentran actualmente en procesos de validación y liberación en la región de Centromérica y El Caribe. La variedad Tío Canela-75, liberada en Honduras en 1996, fue liberada en El Salvador (mayo del 2,000) como "CENTA- 2000", y próximamente será liberada en Nicaragua como "INTACanela". Tío Canela-75 fue liberada en Panamá como "Rojo Chiricano" en 1999, y se encuentra en proceso de validación en Haití. En julio del 2000 la línea MD 2324 fue liberada en Costa Rica con el nombre de "Bribri”. Entre las líneas en proceso avanzado de validación 
Cuadro 9. Promedios de reacción a enfermedades de las líneas y testigos del ECAR-99.

\begin{tabular}{lccccc}
\hline \multicolumn{1}{c}{ Línea } & Antracnosis & $\begin{array}{c}\text { Mancha } \\
\text { Angular }\end{array}$ & $\begin{array}{c}\text { Mosaico } \\
\text { Dorado }\end{array}$ & $\begin{array}{c}\text { Mustia } \\
\text { Hilachosa }\end{array}$ & Roya \\
\hline EAP9510-1 & 8 & 5 & 3 & 6 & 6 \\
EAP 9508-93 & 6 & 5 & 4 & 5 & 6 \\
UPR 9609-2-2 & 5 & 6 & 4 & 6 & 4 \\
EAP 9510-77 & 5 & 6 & 3 & 6 & 7 \\
DICTA 117 & 6 & 6 & 6 & 6 & 6 \\
EAP 9509-29 & 6 & 7 & 5 & 5 & 7 \\
SRC 1-1-18 & 3 & 6 & 5 & 5 & 4 \\
DICTA 146 & 7 & 6 & 5 & 6 & 7 \\
PTC 9558-17 & 5 & 6 & 6 & 5 & 5 \\
PTC 9557-98 & 5 & 6 & 5 & 5 & 5 \\
PM 9422-3 & 8 & 7 & 5 & 5 & 5 \\
Test. Local & 7 & 6 & 7 & 5 & 8 \\
PCE 9351-8 & 6 & 6 & 5 & 5 & 7 \\
SRC 1-12-1 & 8 & 6 & 2 & 6 & 3 \\
Dorado (T.U.) & 8 & 6 & 5 & 6 & 7 \\
SRC 1-18-1A & 6 & 6 & 6 & 5 & 6 \\
& & & & & \\
Promedio & 6 & 6 & 5 & 5 & 6 \\
Localidades & 1 & 5 & 3 & 3 & 2 \\
\hline
\end{tabular}

se encuentran MD 30-37 en Cuba, UPR9609-22-2 ("INTA- Nueva Guinea") y SRC1-5-3 en Nicaragua y SRC1-12-1 ("Milenio") en Honduras. Un grupo de líneas tolerantes a altas temperaturas y resistentes a mosaico dorado, adaptadas a zonas bajas de Centroamérica y con buen grano comercial, están siendo evaluadas en varios países de la región.

\section{CONCLUSIONES Y RECOMENDACIONES}

El mejoramiento de frijol de grano rojo ha tenido un avance favorable en los últimos años gracias a la estrecha colaboración de los programas de Zamorano, la Universidad de Puerto Rico, y la red de colaboradores de PROFRIJOL, incluyendo los programas nacionales involucrados y el CIAT. Esta red colaborativa es esencial para futuros logros en el mejoramiento de frijol de grano rojo, y crítica para el progreso de mejoramiento de frijol de grano negro en los próximos años. Las lecciones aprendidas a través del mejoramiento de frijol de grano rojo serán útiles en el mejoramiento de grano negro. Se deberá enfatizar en la búsqueda de mejores fuentes de resistencia a mustia hilachosa y en utilizar eficientemente las fuentes de resistencia a mancha angular, en especial las del reservorio Andino. Es necesario ampliar el número de materiales negros con resistencia a mosaico dorado conferida por el gen $b g m-1$. Se deberán desarrollar líneas rojas y negras con el gen de resistencia $b c 3$ a mosaico común, emplendo las líneas rojas derivadas de Tío Canela-75. En el mejoramiento de frijol negro se recomienda usar líneas élites rojas con los genes $b g m-1$ y $b c 3$, y con tolerancia a altas temperaturas. Para ambos tipos de grano es necesario emplear las fuentes de tolerancia a baja fertilidad, e identificar nuevas fuentes de tolerancia a sequía.

En el futuro será muy deseable ampliar las contribuciones de líneas avanzadas al VIDAC y al ECAR por los programas nacionales. Las líneas que están siendo desarrolladas en varios países a partir de poblaciones tempranas recibidas del CIAT es una alternativa. Otra opción sería que Zamorano y la Universidad de Puerto Rico siguieran la estrategia del CIAT, entregando poblaciones en generaciones tempranas a los programas nacionales para que estos desarrollen líneas a partir de ellas. Con base en las líneas avanzadas que ingresaron al LINAF-99 y a las poblaciones en generaciones intermedias y avanzadas que actualmente están siendo evaluadas por el Zamorano, la contribución de los programas regionales al mejoramiento de frijol de granos rojo y negro Mesoamericano para los próximos años se ve prometedora.

\section{LITERATURA CITADA}

CIAT, 1987. Sistema estándar para la evaluación de germoplasma de frijol. $56 \mathrm{p}$.

EBERHART, S.A.; RUSSELL, W.A. 1966. Stability parameters for comparing varieties. Crop Sci. 6: 36-40.

FAO. 1998. Anuario de producción de 1997. Colección FAO: Estadística $\mathrm{N}^{\circ} 142$, vol. 51, Roma, Italia, 239 p.

ROSAS, J.C.; CASTRO, A.; JIMÉNEZ, J.; GONZALES, J.; SIERRA, F.; HUMPHRIES, S. 1999a. Metodologías participativas para el mejoramiento in situ del frijol común. In: Memoria del Simposio Internacional y talleres sobre Fitomejoramiento Participativo en América Latina y El Caribe: Un intercambio de experiencias. Quito, Ecuador. Program PRGA/CGIAR (en prensa).

ROSAS, J.C.; CASTRO, A. 1999b. Experiencias en la producción artesanal de semilla en Centroamérica (eds.). Memoria del Taller de Producción y Distribución de Semilla de Frijol en Centroamérica, Zamorano, Honduras, 2000. Escuela Agrícola Panamericana/ Zamorano, Honduras. $101 \mathrm{p}$.

SINGH, S.P. 1999. Improvement of small- seeded race Mesoamerica cultivars. In: Common Bean Improvement in the Twenty-first Century, S.P. Singh (ed.). Kluwer Acad. Publ., Londres. pp 255-274. 